\title{
Application of the effective formula of growth functional to quantitative description of growth of plant cells
}

\author{
Paweł Zajdel $^{1} \cdot$ Mariusz Pietruszka $^{2}$ - Aleksandra Haduch-Sendecka ${ }^{2}$
}

Received: 16 February 2016/Revised: 25 May 2016/Accepted: 27 July 2016/Published online: 5 August 2016

(C) The Author(s) 2016. This article is published with open access at Springerlink.com

\begin{abstract}
An effective formula describing expansive plant growth is derived from the modified Lockhart/Ortega-type equation. Its applicability is demonstrated on selected experimental data extracted from available literature. Quantitative information about the "diffusion rate" $\left(k_{2}\right)$ of the growth factors is obtained for two different model species in plant science: Arabidopsis thaliana L. belongs to the dicots and Zea mays L. belongs to the monocots. It is shown that the value of the diffusion rate may be useful in comparing different datasets and serve as a measure of reproducibility of standard measurements. Analysis of the formula and fits allows to identify and suggest a set of criteria for reporting future experiments, which would improve comparability and reproducibility of the results.
\end{abstract}

Keywords Auxin · Arabidopsis thaliana L. · Juglone · Fusicoccin · Garlic extract - Zea mays L. · Lockhart equation · Ortega equation

Communicated by U. Feller.

Electronic supplementary material The online version of this article (doi:10.1007/s11738-016-2233-4) contains supplementary material, which is available to authorized users.

Mariusz Pietruszka

mariusz.pietruszka@us.edu.pl

1 Institute of Physics, University of Silesia, 40007 Katowice, Poland

2 Faculty of Biology and Environment Protection, University of Silesia, 40032 Katowice, Poland

\section{Introduction}

The modulation of mechanical properties during expansive growth is a hot topic for plant cell growth community (e.g., recently Boudon et al. 2015; Bidhendi and Geitmann 2016 but also in Rojas et al. 2011 and in Barbacci et al. 2013). The physical mechanism of individual cell growth and elongation has been attributed over the last few decades to various factors ranging from wall plasticity concept by Schopfer (2001) through the loss of stability model propagated by Wei and Lintilhac (2007) and Lintilhac (2014, Fig. 1) to the hydrodynamic model propagated by Zonia (2010). A recent review by Kutschera and Niklas (2013) indicated that these two contrasting views (i.e., "plasticity" vs. "instability" model) do not differ in terms of the fundamental mechanism. Indeed, in both cases cell elongation is related to difference between turgor pressure $P$ and a yield threshold $Y$, which in the first case leads to a "creep" growth (Cogsrove 1985, 2000 for review) parameterized by Lockhart (1965) equation $\mathrm{d} V / \mathrm{d} t \sim(P-Y)$ or to an instability in the latter. It must be noted that both approaches fail to describe analytically shapes of growth functions. On the other hand, a recent study on Escherichia coli response to an osmotic shock (Rojas et al. 2014) has shown that in the case of relatively thin $3 \mathrm{~nm}$ bacteria cell walls, the growth rate is not directly dependent on turgor pressure. The results pointed to the cell wall as a limiting factor and reduced turgor pressure as a possible trigger of a signaling cascade (compare with the "recurrent model", Pietruszka 2016). This paradigm change has yet to be confirmed for cell walls of plants, which are much thicker $\sim 100 \mathrm{~nm}$.

However, some explanation of this dichotomy has already been provided by Kroeger et al. (2011) by analyzing pollen tube oscillations, which take place on a similar time scale (minutes) as the time shock response of 
bacteria. Based on experimental data and an analytical model they proposed a self-regulatory mechanism between cell expansion driven by the turgor pressure and opening of calcium channels in a tube apex, which takes place above a certain critical expansion rate. In this context, based on the Lockhart equation, it was shown that for short periods of oscillations, the average growth rate was insensitive to turgor pressure but remained dependent on it for longer oscillations. At least from a physical point of view, considering the steady growth as a limit when (1) the oscillation period goes to infinity and (2) there are no sudden external stimuli, the first paradigm is restored. The oscillations of tube elongation were also observed in the case of the second model for tip-growth studies-root hairs (Marzec et al. 2015). These oscillations were related to polymerization of actin microtubules which occurs periodically (Vazquez et al. 2014).

In the search for a more flexible function, the Lockhart equation has been augmented by Ortega (1985) to account for time variations of the turgor pressure $P(t)$ by addition of $\mathrm{d} P / \mathrm{d} t$ factor. In such terms, it is (at least analytically) possible to bridge the gap between the earlier contrasting views by envisaging an almost constant (or slowly creeping) average value of $P(t)$, with fluctuations $\delta P(t)$ that exceed the "instability" threshold for a short time (Pietruszka and Haduch-Sendecka 2015). In other words, the wall plasticity model would be adequate for longer periods, while cell instability would be confined to the time scales of the fluctuations.

The Ortega equation has proved its flexibility throughout the last decades with the latest amendments taking into account: transpiration and water uptake (Geitmann and Ortega 2009), parameterization of growth factor concentration (Pietruszka 2013) and inclusion of environmental conditions (Barbacci et al. 2013).

One may ask the question, if solutions of the augmented Lockhart/Ortega equation can be used to describe the growth function of the cell or an elongating coleoptile segment and possibly give us more insight into the longterm mechanism of the cell elongation. There seems to be no doubt that on a scale of hours or days, the growth rate is limited by cell wall biosynthesis (see review of Boyer 2009), which is dependent on $(P-Y)$ and the plasticity of the cell wall (Kutschera and Niklas 2013). However, it must be noted that the Lockhart/Ortega approach is not suitable for description of the growth of the whole plant, which must include cell division, differentiation and expansive growth and lies beyond a simple analytical formula (see Boudon et al. 2015 or Bidhendi and Geitmann 2016).

Therefore, neglecting possible short-term fluctuations, and considering $(P-Y)$ to be almost constant (or slowly decreasing-we assume concomitant water uptake),
Winship et al. (2010), one can justify the use of the Lockhart and Ortega formalism but expanded to include growth factor concentration (Pietruszka 2012). Under such conditions, it should be possible to obtain an approximate solution of the Lockhart/Ortega equation in the long time regime, which would primarily be based on the plasticity model. However, the potential agreement of a formula derived from the plasticity model, would not automatically nullify the "instability" paradigm, as they act on different time scales.

In its basic application, such a formula would allow to compare and contrast experimental results without favoring any of the phenomenological models. However, in its deeper interpretation, it may be used to quantify growth functions using parameters directly connected to the cell wall plasticity like a concentration of different growth factors. For example, it can be used to compare growth curves under different experimental conditions by describing them by a set of fittable parameters, which later on can be interpreted in the context of cell wall biosynthesis. This formula might be also used in comparative studies of different genotypes, i.e., wild-type and mutant plants, to describe function of mutated gene in the processes of cell growth. During last few years the significant progress in description of molecular basis of cell elongation was made, but there is still lack of precise methods for quantification and modeling of plant cell growth.

\section{Methods}

The derivation is based on an earlier notion (Pietruszka 2012) that a growth factor (WLF) concentration formula can be obtained from a sum of growth factor production rate $k_{1}\left(\left[k_{1}\right]=\right.$ concentration $\bullet$ time $\left.{ }^{-1}\right)$ and a depletion-like part with proportionality constant $k_{2}\left(\left[k_{2}\right]=\right.$ time $\left.^{-1}\right)$ :

$\frac{\mathrm{d} n(t)}{\mathrm{d} t}=k_{1}-k_{2} n(t)$,

hereafter referred to as "diffusion rate" (note, it should not be confused with the "diffusion constant" $D$ in the Fick's law, for no spatial gradients were introduced). One can think of $k_{2}$ in terms of depletion and diffusion since both processes contribute to the rate of change of $n(\mathrm{t})$ proportionally to $n(\mathrm{t})$.

One has to note that similar but much complicated and more specific models have already been introduced in the literature. For example, Rojas et al. (2011) (see Eq. 2) used similar terms to describe the kinetics of pectin chemistry in the oscillatory growth of pollen tube cell. In this context $k_{1}$ would be an equivalent of the de-esterification and $k_{2}$ would incorporate cross-linking, dilution by deposition and advection terms. In our case, we would not want to limit 
the formula to any specific factors like calcium pectates (Proseus and Boyer 2006) or hormones (Chavarria-Krauser et al. 2005). Therefore, the derivation presented here is detailed but generally applicable (see Supplementary Information Eqs. (1)-(14) for detailed discussion) and after several steps one obtains general solution in form:

$V(t)=V_{0} \mathrm{e}^{\Phi_{0} n_{0} \frac{1}{k_{2} k_{0}}\left(t+\left(\frac{1}{k_{2}}-\frac{n_{0}}{k_{1}}\right)\left(e^{-k_{2} \cdot t}-1\right)\right),}$

where $V$ stands for the volume evolving in time $t$, $V_{0}=V(t=0)$, and $\Phi_{0}$ represents the Lockhart constant (representing viscoelastic properties of the cell wall; $\left[\Phi_{0}\right]=10^{-6} \mathrm{MPa}^{-1} \times \mathrm{s}^{-1}$ ). The formula can be simplified (see SI Eqs. (15)-(22)) by considering only time intervals shorter than growth factor production rate $k_{1}$, so $k_{1} t<1$, which decouples the equation into two major contributions. The first component (SI Eq. (18)) is primarily dependent on the production rate $k_{1}$ and can be represented by simple linear term $A t+B$. The second one [SI Eqs. (19)-(20)] is dominated by the depletion-like effects $\left(k_{2}\right)$ and has a form of double exponent $\exp \left(-\exp \left(-k_{2} t\right)\right)$; its representation, using parameters $\mathrm{C}$ and $\mathrm{D}$, has been described in the last paragraph of the Supplementary Information (SI).

We shall neglect the correlations between above terms, and simply add both contributions arriving at the formula derived from an approximated solution of the modified Lockhart/Ortega equation:

$\frac{V(t)-V_{0}}{V_{0}}=A t+B+C e^{-e^{-D(t-t e)}}$

Equation (3) describes the relative growth curves and is a simple sum of a linear "start-up" region and a nonlinear accelerated (and decelerated) growth. The two time regions are both valid in the long time scale in which the plasticity mode is dominant (see "Introduction"). Here, we introduced parameter $\mathrm{t}_{\mathrm{e}}$ (effective start-up time) to deal with mathematically and practically inseparable contributions from (1) multiplicative factor "F" (see SI) in front of the inner exponent and (2) difficult to estimate moment when plant cell or elongating organ resumes growth. In cases when a growth factor is added to the plant environment, $t_{e}$ cannot be directly equated with the time of addition but rather as an effective moment at which it starts to dominate. The indeterminate starting point also affects the linear part $A t+B$ and any time shift $A \cdot \mathrm{d} t$ is included in the parameter $B$. Additionally, due to approximations made in the SI Eq. (18) and SI Eq. (20), we introduced parameters $B_{1}$ and $B_{2}$ as any offsets of the (relative) elongation at $t=0$, including slowly varying (almost constant) terms in the expansion, noise and measurement uncertainty. To sum up, parameter $B$ includes all constants and slowly varying orders of expansion and therefore has no theoretical use and effectively normalizes the result to the first data point. From the experimental side, it accounts for any constant offset of the experimental data, for example whenever length is used instead of a relative elongation (see Fig. 2) or for uncertainty in a determination of $V_{0}$. For practical purposes and dimensional consistency it might also be convenient to use time constants $T_{1} \sim 1 / k_{1}$ and $T_{2} \sim 1 / k_{2}$ instead of production and depletion rates $k_{1}$ and $k_{2}$, respectively (for the detailed description see SI).

Fitting this equation to the experimental data provides parameters $A, B, C, D$ and $t_{e}$ which later on are connected with the $\bar{\Phi}_{0}$ and $T_{2}$, under a condition that the proper unit scaling was done (for example, if the experimental data contains relative elongation in $\mu \mathrm{m} / \mathrm{cm}$, it must be corrected by a factor of $1 / 10,000)$. It has to be stated that dropping the assumption made earlier that $T_{1} \gg T_{2}$ would mostly change B and C, and only weakly affect $\bar{\Phi}_{0}$ and $T_{2}$ (note that $A, B$ and $C \geq 0$ ).

Here, we can already identify at least one parameter of the equation $\mathrm{D}\left(k_{2}\right)$ as $1 / T_{2}$ and get a first estimate of $\bar{\Phi}_{0}$ from parameter $A$. At the current approximation level, parameter $C$ can only be used to quantify growth as an equivalent of 'growth amplitude' (for further interpretation, in the context of "acid growth", see e.g., Pietruszka and Haduch-Sendecka 2016). Following the convention used in SI Eq. (20), $C$ can be roughly associated with $k_{2}\left(1 / T_{2}\right)$ through $C \sim \exp \left(T_{2}\right) \sim \exp \left(1 / k_{2}\right)$ but it would be valid only in the epoch when a diffusion mechanism is dominant (nonlinear). It needs emphasizing that the formula describes the (relative) change of the volume, whereas the experimental data is usually given as relative elongation or length increments, neglecting any changes in diameter. However, such approximation only weakly affects the value of parameter $D\left(k_{2}, T_{2}\right)$ obtained from the fit. It follows from the fact that $D$ comes from the double exponential term $\exp \left(-\exp \left(-D\left(t-t_{e}\right)\right)\right)$, which can accumulate any cube or square root operations in the parameter $t_{e}$.

Finally, in order to obtain other parameters $\left(\bar{\Phi}_{0}, T_{1}\right)$ we would have to use the complete formula.

$\left.V(t)=V_{0} e^{-\bar{\Phi}_{0} \frac{\mathrm{T}_{2}}{\mathrm{~T}_{1}}\left[\left(\mathrm{~T}_{1}-\mathrm{T}_{2}\right)\right.}\left(e^{\frac{-t}{\mathrm{~T}_{2}}}-1\right)-t\right]$

which is highly nonlinear and not practical for common applications. In other words, the (semi-empirical) formula allows fitting of $T_{2}$ and estimation of $\bar{\Phi}_{0}$, which is related to $\Phi_{0}(P-Y) \mathrm{n}_{0}$, where $\Phi_{0}$ is a Lockhart constant, $(P-Y)$ difference in pressures. The proportionality factor $\mathrm{n}_{0}$ comes from the unknown initial concentration of growth factors at $t=0$. Within the current approximation $A \sim \Phi_{0}(P-Y) n_{0}$, and $A$ should be dependent on concentration $n_{0}$. 
In order to test the applicability of the formula, a computer program written in Python (Oliphant 2007) programming language was prepared (Zajdel et al. 2014). Its functionality comprises three parts: (1) reading and scaling of the data; (2) obtaining an initial estimate of fit parameters; (3) fitting procedure using Simplex (Nelder-Mead algorithm) and least squares (Levenberg-Marquardt LSQ) methods implemented in package scipy.optimize (van der Walt et al. 2011; Jones et al. 2001).

Part (1) aims to ensure that the fitting parameters have proper dimensions and requires the $y$-axis to be dimensionless relative elongation and the $x$-axis-time in seconds. If the above conditions are met, parameter A contains contribution from $\Phi_{0}(P-Y) \mathrm{n}_{0}$ and parameter $\mathrm{D}$ is equal to $k_{2}\left(\mathrm{~s}^{-1}\right)\left(T_{2}\right.$ is expressed in seconds). If the incoming data are not normalized, then $T_{2}$ is expressed in time units of the original measurement.

Part (2) utilizes user's interaction (point-and-click on screen) to obtain an estimate of $t_{\mathrm{e}}$, which is roughly equivalent to the inflection point of the curve. Then, an initial fit is performed using Simplex algorithm, which is slow but stable at this level of the refinement, when only initial estimates of parameters are known. As the last step, a full matrix least square fit is applied in order to estimate standard deviations of the parameters. We have to note that a successful fit of the LSQ part requires data of good quality, which meet criteria given later in the paper. The visualization of fits is preformed using the Matplotlib package (Hunter 2007).

\section{Results}

The practical use of the formula was carried out on several growth curves which were collected from available figures and converted to data points from a literature survey.

The experimental base included:

1. Arabidopsis thaliana L. (Boyes et al. 2001, Fig. 3C), (Gendreau et al. 1997, Fig. 1A, B), (Nishimura et al. 2004, Fig. 4A),

2. Zea mays L. (Schopfer 2006, Fig. 2A), (Pietruszka 2010, Fig. 7A, B), (Polak et al. 2012, Figs. 2, 3, 8), (Rudnicka et al. 2013, Figs. 1, 2, 5).

At present, the only parameter that can be directly inferred from the fit is the diffusion rate $k_{2}$, which involves the effective transport to/from the cell wall. Tables 1, 2, 3 and 4 and SI Tables 1-10 present diffusion rates obtained from fits for the selected datasets. The results are presented in seconds as a common SI unit. For a better comparison, a second column gives the results in the time units used in each paper. Before the fits the data were normalized in the fitting software to the dimensionless quantities, but the figures were plotted in the original measured units.

Examples of selected fits are presented in Figs. 1 and 2. For interested readers, a complete set of figures was provided in the Supplemental Information (SI) file as SI Figs. 1-10. All of the plots show linearly interpolated experimental points together with both components of the equation presented on a denser grid: the linear growth (linear) and nonlinear diffusive term (nonlinear). The vertical olive line marks the fitted value of $t_{\mathrm{e}}$. The estimated statistical uncertainties (standard deviations) of the parameters were obtained from the LSQ part of the fits and were reported on $1 \sigma$ level.

\section{Discussion}

The discussion of the results will be divided into two parts. First, an applicability of the formula will be checked. Later on, a general comparability of results between different experimental conditions and species will be reviewed.

\section{Applicability of the formula}

The formula SI Eq. (20) was tested on two plants Zea mays L. and Arabidopsis thaliana L. using 39 datasets digitized from 7 papers, which came from 6 different measurement methodologies. The fits to the growth (or relative growth) curves were very good in 31 cases and good in 4 [SI Fig. 1B-3 (open and closed circles in the original data)]. One fit showed discrepancy at early time [SI Fig. 4 (plus sign)]. In the remaining 3 cases (SI Fig. 5 diamond, SI Fig. 7 closed and open triangle) the fit converged to solutions with a wrong slope of the linear part. We have to note that the good agreement in almost $90 \%$ cases is obtained despite the fact that we are only analyzing length (relative elongation) instead of the relative volume.

The decrease of the general agreement in the 7 unsatisfactory cases can be related to the time range of the reported data. The initial linear "lag" term of the formula requires that a sufficiently long time span at the beginning of the data set is measured. The second nonlinear sigmoid curve must be measured at least to its inflection point and preferably have a reasonable "follow-up" at the end of the measurement.

Especially the first condition must be fulfilled as it gives a stable base line for the nonlinear part. Omission of this initial region may potentially hinder successful fits to the data obtained in the usually time consuming experiments. The short length of the start-up region versus the postgrowth saturation biases the fit of the linear part towards the post-growth slope. The bias causes fit to be worse at an onset of the nonlinear part which is seen on several plots 
Table 1 Diffusion rate obtained for coleoptile of maize grown under constant dim green light at $25^{\circ} \mathrm{C}$ and influence of indole-3-acetic acid (IAA) and fusicoccin (FC)

\begin{tabular}{lllrr}
\hline Polak et al. 2012 & $k_{2}\left(\mathrm{~s}^{-1}\right)$ & $k_{2}\left(\mathrm{~min}^{-1}\right)$ & $T_{2}(\mathrm{~s})$ & $T_{2}(\mathrm{~min})$ \\
\hline Figure 2: black diamond, control & $(12.6 \pm 0.6) \mathrm{e}-05$ & $0.0076 \pm 0.0003$ & $7920 \pm 360$ & $132 \pm 6$ \\
Figure 3: black triangle, IAA $10^{-5} \mathrm{M}$ & $(29.3 \pm 1.5) \mathrm{e}-05$ & $0.0176 \pm 0.0009$ & $3410 \pm 170$ & $56.9 \pm 2.9$ \\
Figure 8: black triangle, FC $10^{-6} \mathrm{M}$ & $(18.6 \pm 2.8) \mathrm{e}-05$ & $0.0112 \pm 0.0017$ & $5370 \pm 810$ \\
\hline
\end{tabular}

Table 2 Fit parameters for coleoptile of maize grown under constant dim green light at $25{ }^{\circ} \mathrm{C}$ and influence of indole-3-acetic acid (IAA) and fusicoccin (FC)

\begin{tabular}{lllll}
\hline Polak et al. 2012 & $A\left(\mathrm{~s}^{-1}\right)$ & \multicolumn{1}{l}{$B$} & \multicolumn{1}{l}{$D$} & $\left(\mathrm{~s}^{-1}\right)$ \\
\hline Figure 2: black diamond, control & $(1.4 \pm 0.1) \mathrm{e}-06$ & $0.0017 \pm 0.0006$ & $0.0872 \pm 0.0048$ & $(12.6 \pm 0.6) \mathrm{e}-05$ \\
Figure 3: black triangle, IAA $10^{-5} \mathrm{M}$ & $(2.7 \pm 0.1) \mathrm{e}-06$ & $-0.0023 \pm 0.0008$ & $0.0985 \pm 0.0038$ & $(29.3 \pm 1.5) \mathrm{e}-05$ \\
Figure 8: black triangle, FC $10^{-6} \mathrm{M}$ & $(3.6 \pm 1.1) \mathrm{e}-06$ & $-0.0117 \pm 0.0037$ & $0.1870 \pm 0.0360$ & $(18.6 \pm 2.8) \mathrm{e}-05$ \\
\hline
\end{tabular}

Table 3 Diffusion parameter obtained from natural growth cycle of wild (Col-0) Arabidopsis measured during principal growth stages 5 and 6

\begin{tabular}{lllll}
\hline Boyes et al. 2001: Fig. 3C & $k_{2}\left(\mathrm{~s}^{-1}\right)$ & $k_{2}\left(\mathrm{day}^{-1}\right)$ & $T_{2}(\mathrm{~s})$ & $T_{2}(\mathrm{day})$ \\
\hline Wild-type Col-0 & $(1.3 \pm 0.3) \mathrm{e}-06$ & $0.11 \pm 0.02$ & $800000 \pm 170000$ & $9.3 \pm 1.9$ \\
\hline
\end{tabular}

Table 4 Fit parameters for natural growth cycle of wild (Col-0) Arabidopsis measured during principal growth stages 5 and 6

\begin{tabular}{lllll}
\hline Boyes et al. 2001: Fig. 3C & $A\left(\mathrm{~s}^{-1}\right)$ & $B$ & $C$ & $D\left(\mathrm{~s}^{-1}\right)$ \\
\hline Wild-type Col-0 & $(0.0 \pm 2.1) \mathrm{e}-09$ & $0.0046 \pm 0.0006$ & $0.0316 \pm 0.0094$ & $(1.3 \pm 0.3) \mathrm{e}-06$ \\
\hline
\end{tabular}

[especially on SI Fig. 5 (bottom right) and SI Fig. 7 (middle)].

We have to point out that the obtained diffusion rates cannot be directly equated to the growth rate. A good example can be drawn by comparison of the results in SI Table 1, where the diffusion rate $k_{2}$ (parameter $\mathrm{D}$ ) is slightly higher for the hypocotyls grown in the light than those grown in the dark. Here, over tenfold increase in length is incorporated into amplitude parameter $\mathrm{C}$. Nonetheless, this result has its own interesting interpretation and reference to the literature; well known is the fact that light diminishes the level of active auxin in irradiated plant organs and induces its diffusion into shade areas; the increase in the depletion rate $k_{2}$ exactly and numerically reflects this phenomenon.

Hereafter, we shall only concentrate on the parameter $k_{2}$ (D) as the one connected with the microscopic properties of the model. Its usability is apparent in two areas:

1. A higher value of $k_{2}$ is equivalent to a faster depletion rate of the growth factor concentration with the lower limit reached at the linear follow-up region at the end of the measurement.

2. Under the same growth conditions plants should display the same diffusion rates, which should give a unique opportunity to verify reproducibility and compare different measurements. It should also aid in predicting values of $k_{2}$ in future experiments (interpolation between known cases or close extrapolation).

\section{Parameterization of the experimental data}

The comparison of different diffusion rates could be safely made only in a few cases where authors reported several of parameterized datasets. The results are presented in SI Table 2 (Nishimura et al. 2004), SI Tables 5-7 (Polak et al. 2012) and SI Tables 8-10 (Rudnicka et al. 2013). The diffusion rates and amplitude parameters $(C)$ for each table were collected and presented, respectively, in Fig. 3 for Nishimura et al. (2004), SI Figures: 11, 12, 13 for Polak et al. (2012), SI Figures: 14, 15, 16 for Rudnicka et al. (2013). 

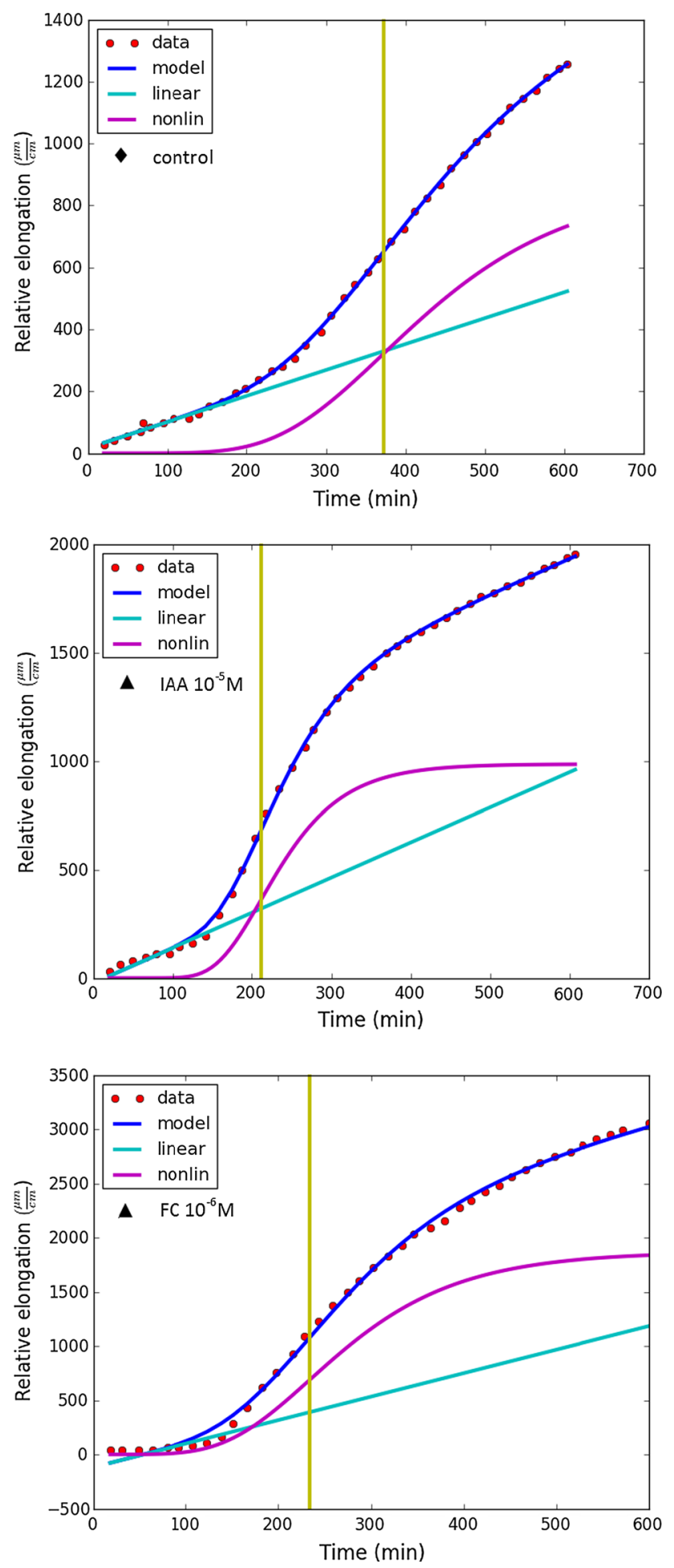

The results inferred from the paper of Nishimura et al. (2004) presenting growth rate of Arabidopsis thaliana L. mutants in single $A H K$ genes (encoding Arabidopsis Histidine Kinase cytokinin receptors), show similar values of calculated $k_{2}$ for both wild-type ecotypes (Columbia and Wassilewskija) and a systematic increase of the calculated
4Fig. 1 Fit to the data of Polak et al. (2012) describing growth for coleoptile segments of maize under constant dim green light at $25{ }^{\circ} \mathrm{C}$ in control conditions (closed diamond in Fig. 2) and under the influence of indole-3-acetic acid (IAA $10^{-5} \mathrm{M}$-closed triangle in Fig. 3) and fusicoccin (FC $10^{-6} \mathrm{M}$-closed triangle in Fig. 8). Compare with Lüthen et al. (1990) or Hager (2003) in the general context of "acid growth hypothesis", and the results presented in SI Figs. 11-13 for the estimated diffusion rate $k_{2}$ and amplitude $C$ (see also Pietruszka and Haduch-Sendecka 2016). The biosynthesis (production) linear region ends up approximately at $t_{e}$, while the curvilinear (inactivation) region prevails above $t_{e}$

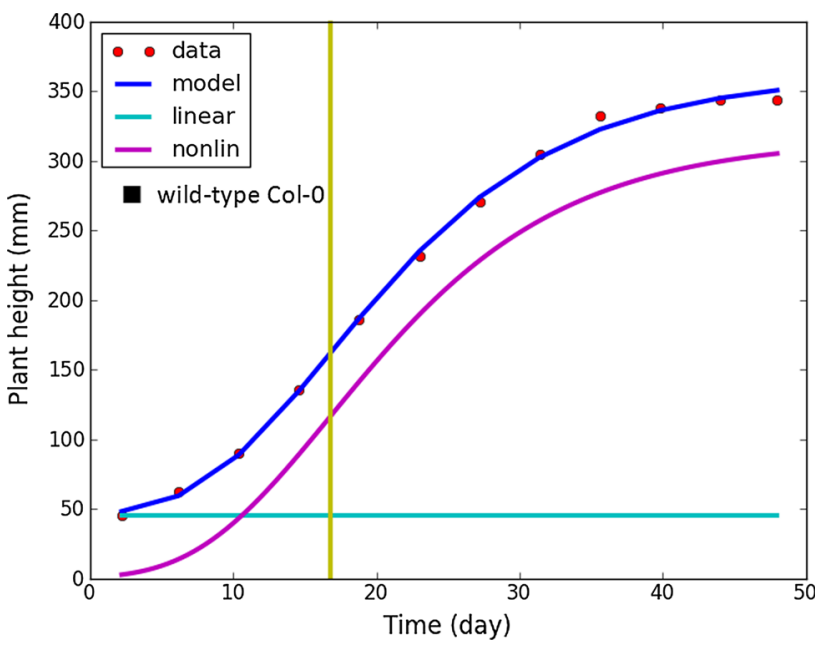

Fig. 2 Fit to the data of Boyes et al. 2001 (Fig. 3c) describing natural growth cycle of wild (Col-0) Arabidopsis thaliana $\mathrm{L}$.

diffusion rate for subsequent mutations (Fig. 3). A quantitative analysis of the plot indicates that $k_{2}$ was significantly affected in plants containing ahk3-1 mutations (2 double mutants and the triple one). The double mutant ahk2-1 ahk4-1 had a diffusion rate similar to the both analyzed wild-type ecotypes, which points at ahk3-1 as the critical factor. Here, one can also qualitatively verify an approximate dependence $C \sim \exp \left(1 / k_{2}\right)$ since $C$ decreases as $k_{2}$ increases (SI Eq. 22).

A wider comparison can be made on the basis of papers reporting growth kinetics of Zea mays L. Polak et al. (2012) and Rudnicka et al. (2013). In the first one a garlic extract (GE) containing thiosulphinate was used as a growth inhibitor $(4.4 \pm 0.3 \mathrm{mM})$ in solutions diluted 50 , 20, 15 and 10 times. SI Fig. 11 presents dependence of the diffusion parameter $k_{2}$ obtained under different dilutions of GE. Both $10 \times$ and $50 \times$ solutions display remarkably similar values of $k_{2}$, which are larger (faster depletion) than values obtained from control growth. The value for $20 x$ dilution is on the other hand much lower even than the control, which can be either an outlier or result from a systematic trend. This becomes more clear by comparison of SI Figs. 12 and 13, which contain fit results for systems 


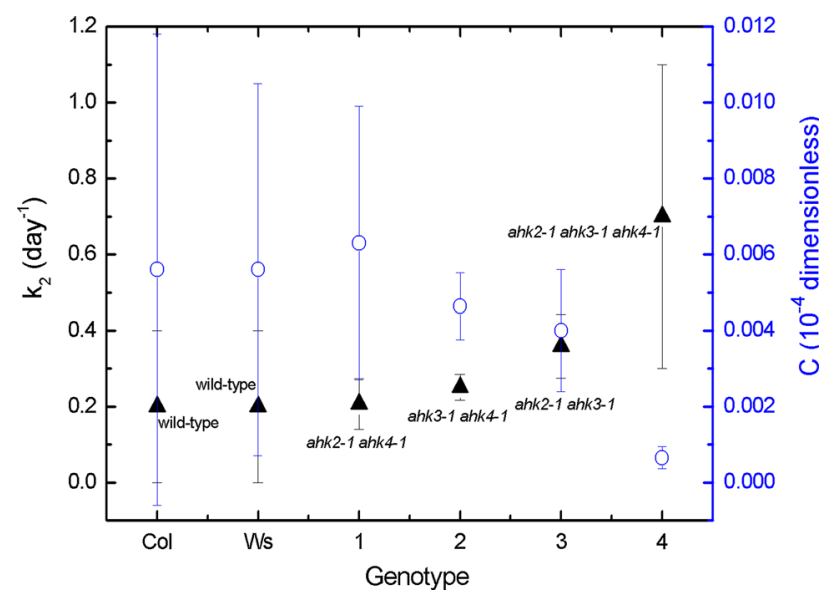

Fig. 3 Diffusion rate $k_{2}$ and amplitude $C$ for roots of Arabidopsis thaliana L. grown under constant lighting conditions, corresponding to the fit in SI Fig. 2. The error bars represent statistical uncertainty on $1 \sigma$ level obtained from the LSQ part of the fit (std-dev)

with indole-3-acetic acid (IAA) and fusicoccin (FC). The results suggest that between $10 \times$ and $20 \times$ dilution there is a systematic decrease of the $k_{2}$ factor, which requires further investigation.

The next set of papers studied growth properties of Zea mays L. under influence of juglone (JG) and IAA. The JG itself significantly changed growth kinetics at $10 \mu \mathrm{M}$ concentration, where the nonlinear part was almost completely suppressed. Under increased concentration of JG the $k_{2}$ values came closer to the control, which was rather unusual behavior (SI Fig. 14).

On the other hand, a clear trend could be identified in the $100 \mu \mathrm{M}$ IAA + JG series (SI Fig. 15), where addition of $\mathrm{JG}$ led to increased values of $k_{2}$ indicating an increased depletion rate. At the same time, the amplitude $C$ decreased. Similar tendency was found in the JG + FC case (SI Fig. 16).

\section{Comparison and transferability of results between papers}

Comparison of growth kinetics of plants depends on multiple factors: plant genetics, incubation medium, lighting and temperature conditions just to name a few. As a result the comparison of the papers and reproducibility between groups utilizing different experimental approaches is a very difficult task. At least one has to remain consistent within its own technique.

The results obtained in the first section (Arabidopsis thaliana L.) were barely comparable as they differed in light cycling used for growth. The values of $k_{2}\left(\mathrm{~s}^{-1}\right)$ obtained for natural growth $1.2 \times 10^{-6}$ (Boyes et al.

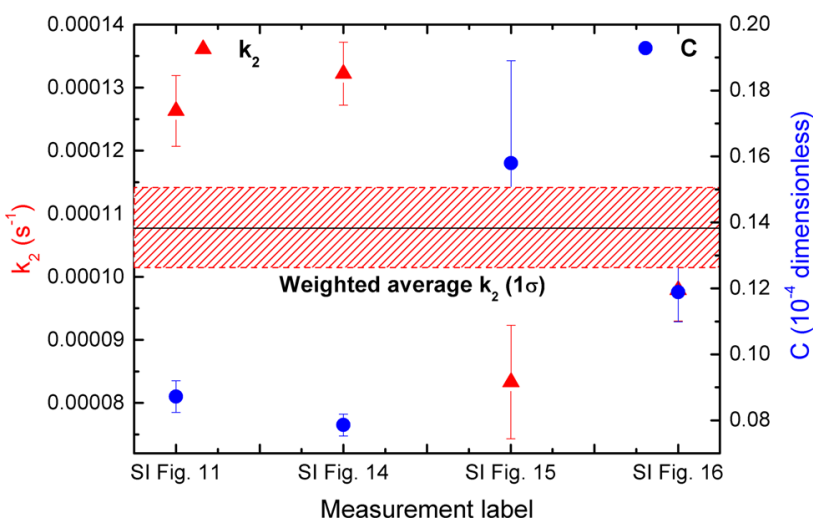

Fig. 4 Diffusion rate $k_{2}$ and amplitude parameter $C$ obtained for Zea mays L. grown under constant green light at $25{ }^{\circ} \mathrm{C}$. Error bars depict statistical uncertainty on $1 \sigma$ level

2001), $16 \mathrm{~h}$ light and $8 \mathrm{~h}$ dark $1.2 \times 10^{-5}$ (Gendreau et al. 1997) or constant light $2.5 \times 10^{-6}$ (Nishimura et al. 2004) differed by an order of magnitude (growth temperatures were not comparable as well).

The better situation was in the case of Zea mays L. with one reported case of dark grown plants (Schopfer 2006) with $k_{2}=0.90(2) \mathrm{s}^{-1}$ and four grown under constant green light at $25{ }^{\circ} \mathrm{C}$ (Polak et al. 2012; Rudnicka et al. 2013). Figure 4 presents results collected from SI Tables 5 and $8-10$. There is a reasonable agreement between the control values, which are equal within $4 \sigma$ limit and the estimated weighted average is $(1.08 \pm 0.06) \times 10^{-4}$ (hatched area). This might be used as a guide for future experiments allowing to reject outliers (due to faulty conditions) already at the control stage, thus avoiding wasting resources.

\section{Suggested set of experiment criteria required for a successful fit using Eq. (4)}

The applicability of the semi-phenomenological formula to a fit requires data of sufficient quality. Here is a suggested minimal set of indications required for a good fit:

1. A sufficiently long, almost linear, 'start-up' phase must be recorded at the beginning of the data collection. Data containing 'kinks' or delayed effects must be rejected at this early stage. (For example, SI Figs. 3, 4 (asterisk), 7 (open triangle), 8 (semi-open diamond) with fits to data with insufficient start-up region.)

2. The nonlinear part must be fully presented from the bottom to top bends.

3. The 'follow-up' linear part cannot be too long (longer than the start up). It can be measured, but must be shortened for the fit. First of all-it lies beyond the approximation region $\left(t<T_{2}\right)$. Secondly, its slope will 
dominate in the fit of the linear contribution to the formula causing bad agreement in the early times.

The above criteria consider only a technical aspect of fit, that is a stable numerical solution. They are not aimed to guarantee the overall correctness of the result. Also when additional growth factors are used, the addition time should not coincide with the onset of the nonlinear part (e.g., as results in SI Fig. 10 after Rudnicka et al. 2013) since it would result in an unsettled 'shock' state (unless that is the intention of the operator). When modifications of growth medium are performed, a stability of the growth conditions must be assured.

\section{Conclusions}

The connection between the experiment and theory of the plant enlargement by cell elongation was done on the basis of a modified Lockhart/Ortega kind of equation linking the diffusion parameter $k_{2}$ with the microscopic properties of the biophysical model. The derived Eq. (3) allows to quantitatively describe the shape of a growth curve for plants as was shown for model species Arabidopsis thaliana L. and Zea mays L. The obtained values allowed for deeper analysis of growth kinetics allowing to clearly identify trends and abnormalities in the growth curves. Recently, the presented approach was successfully applied to quantitatively describe effective diffusion rates and correlations between growth and proton influx rates in Zea mays L. (Pietruszka and Haduch-Sendecka 2016).

The transferability (comparability and reproducibility) of diffusion parameter $k_{2}$ has been demonstrated in the case of Zea mays L. grown under the same experimental conditions, where the weighted average value was found to be $(1.08 \pm 0.06) \times 10^{-4}$.

Further expansion of the approach into new species and diverse conditions of growth might aid in creating a database of biophysical parameters for different plants. In the future, this cumulative knowledge may help in inferring valuable new results without experimental work.

To sum up, the simple formula can be used to fit a vast number of datasets for different plants and under variable conditions. The quantitative character of the fits: (1) provides researchers with new opportunities to analyze and interpret their data; (2) gives a better chance to catch and scrutinize outliers and (3) allows to focus on areas of interest, which would be impossible with only qualitative comparison. The above factors, can be used to open up new avenues in analyzing growth curves of plants and organs.

Author contribution statement PZ and MP conceived and wrote the paper. PZ wrote the computer code (Python). AH-S: data acquisition and analysis.

\section{Compliance with ethical standards}

Conflict of interest None of the authors have any competing interests in the manuscript.

Open Access This article is distributed under the terms of the Creative Commons Attribution 4.0 International License (http://crea tivecommons.org/licenses/by/4.0/), which permits unrestricted use, distribution, and reproduction in any medium, provided you give appropriate credit to the original author(s) and the source, provide a link to the Creative Commons license, and indicate if changes were made.

\section{References}

Barbacci A, Lahaye M, Magnenet V (2013) Another brick in the cell wall: biosynthesis dependent growth model. PLoS One 8:e74400

Bidhendi AJ, Geitmann A (2016) Relating the mechanics of the primary plant cell wall to morphogenesis. J Exp Bot 67:449-461

Boudon F, Chopard J, Ali O, Gilles B, Hamant O, Boudaoud A, Traas J, Godin C (2015) A computational framework for 3D mechanical modeling of plant morphogenesis with cellular resolution. PLoS Comput Biol 11:e1003950

Boyer JS (2009) Cell wall biosynthesis and the molecular mechanism of plant cell enlargement. Funct Plant Biol 36:393-394

Boyes DC, Zayed AM, Ascenzi R, McCaskill AJ, Hoffman NE, Davis KR, Görlach J (2001) Growth stage-based phenotypic analysis of Arabidopsis: a model for high throughput functional genomics in plants. Plant Cell 13:1499-1510

Chavarria-Krauser A, Jäger W, Schurr U (2005) Primary root growth: a biophysical model of auxin-related control. Funct Plant Biol 32:849-862

Cogsrove DJ (1985) Cell wall yield properties of growing tissues. Evaluation by in vivo stress relaxation. Plant Physiol 78:347-356

Cogsrove DJ (2000) Loosening of plant cell walls by expansins. Nature 407:321-326

Geitmann A, Ortega JKE (2009) Mechanics and modelling of plant cell growth. Trends Plant Sci 14:467-478

Gendreau E, Traas J, Demos' T, Crandjean O, Caboche M, Hofte H (1997) Cellular basis of hypocotyl growth in Arabidopsis thaliana. Plant Physiol 114:295-305

Hager A (2003) Role of the plasma membrane $\mathrm{H}^{+}$-ATPase in auxininduced elongation growth: historical and new aspects. J Plant Res 116:483-505

Hunter JD (2007) Matplotlib: a 2D graphics environment. Comp Sci Eng 9:90-95

Jones E, Oliphant E, Peterson P et al (2001) SciPy: Open Source Scientific Tools for Python. http://www.scipy.org/. Online; Accessed 24 Mar 2016

Kroeger JH, Zerzour R, Geitmann A (2011) Regulator or driving force? The role of turgor pressure in oscillatory plant cell growth. PLoS One 6:e18549

Kutschera U, Niklas KJ (2013) Cell division an turgor driven stem elongation in juvenile plants: a synthesis. Plant Sci 207:45-46

Lintilhac P (2014) The problem of morphogenesis: unscripted biophysical control systems in plants. Protoplasma 251:25-36

Lockhart JA (1965) An analysis of irreversible plant cell elongation. J Theor Biol 8:264-275

Lüthen H, Bigdon M, Böttger M (1990) Reexamination of the acid growth theory of auxin action. Plant Physiol 93:931-939

Marzec M, Melzer M, Szarejko I (2015) Root hair development in the grasses: what we already know and what we still need to know? Plant Physiol 168:407-414 
Nishimura Ch, Ohashi Y, Sato S, Kato T, Tabata S, Ueguch Ch (2004) Histidine kinase homologs that act as cytokinin receptors possess overlapping functions in the regulation of shoot and root growth in Arabidopsis. Plant Cell 16:1365-1377

Oliphant T E (2007) Python for scientific computing in computing in science and engineering 9:10-20. doi:10.1109/MCSE.2007.58. Python Programming Language Official Website http://www. python.org

Ortega JKE (1985) Augmented growth equation for cell wall expression. Plant Physiol 79:318-320

Pietruszka M (2010) Exact analytic solutions for a global equation of plant cell growth. J Theor Biol 264:457-466

Pietruszka M (2012) A biosynthesis/inactivation model for enzymatic WLFs or non-enzymatically mediated cell evolution. J Theor Biol 315:119-127

Pietruszka M (2013) Special solutions to the Ortega equation. J Plant Growth Regul 32:102-107

Pietruszka M (2016) pH/T duality-equations of state and evolution for plants. Front Plant Sci, In review

Pietruszka M, Haduch-Sendecka A (2015) Pressure-induced wall thickness variations in multi-layered wall of a pollen tube and Fourier decomposition of growth oscillations. Gen Physiol Biophys 34:145-156

Pietruszka M, Haduch-Sendecka A (2016) Effective diffusion rates and cross-correlation analysis of "acid growth" data. Acta Physiol Plant 38(53):1-17

Polak P, Zaborska W, Tukaj Z, Karcz W (2012) Effect of thiosulphinates contained in garlic extract on growth, proton fluxes and membrane potential in maize (Zea mays L.) coleoptile segments. Acta Physiol Plant 34:41-52

Proseus TE, Boyer JS (2006) Calcium pectate chemistry controls growth rate of Chara coralline. J Exp Bot 57:3989-4002
Rojas ER, Hotton S, Dumais J (2011) Chemically mediated mechanical expansion of the pollen tube cell wall. Biophys J 101:1844-1853

Rojas ER, Theriot J, Huang KC (2014) Response of Escherichia coli growth rate to osmotic shock. PNAS 111:7807-7812

Rudnicka M, Polak P, Karcz W (2013) Cellular responses to naphthoquinones: juglone as a case study. Plant Growth Regul $72: 239-248$

Schopfer P (2006) Hydroxyl radical-induced cell-wall loosening in vitro and in vivo: implications for the control of elongation growth. Plant J 28:679-688

Vazquez LA, Sanchez R, Hernandez-Barrera A, Zepeda-Jazo I, Sánchez F, Quinto C, Torres LC (2014) Actin polymerization drives polar growth in Arabidopsis root hair cells. Plant Signal Behav 9:e29401

Walt van der S, Colbert S C and Varoquaux G (2011) The NumPy array: a structure for efficient numerical computation in computing in science and engineering 13:22-30. doi:10.1109/MCSE. 2011.37. Numerical Python Official Website http://www.numpy. org

Wei C, Lintilhac PM (2007) Loss of stability: a new look at the physics of cell wall behavior during plant cell growth. Plant Physiol 145:763-772

Winship LJ, Obermeyer G, Geitmann A, Hepler PK (2010) Under pressure, cell wall sets the pace. Trends Plant Scie 15:363-369

Zajdel P, Haduch-Sendecka A, Pietruszka M (2014) Python code "fitexex-application of $\exp (\exp ())$ function for plant physiology". https://github.com/pawelzajdel/fitexex

Zonia L (2010) Spatial and temporal integration of signalling networks regulating pollen tube growth. J Exp Bot 61:1939-1957 